\title{
The bone marrow compartment is modified in the absence of galectin-3
}

\author{
C. Brand • F. L. Oliveira • L. Ricon • M. L. Fermino • \\ L. C. Boldrini • D. K. Hsu • F. T. Liu $\cdot$ R. Chammas • \\ R. Borojevic • M. Farina $\cdot$ M. C. El-Cheikh
}

Received: 3 May 2011 /Accepted: 17 October 2011 /Published online: 27 November 2011

(C) The Author(s) 2011. This article is published with open access at Springerlink.com

\begin{abstract}
Galectin-3 (gal-3) is a $\beta$-galactoside binding protein present in multivalent complexes with an extracellular matrix and with cell surface glycoconjugates. In this context, it can deliver a variety of intracellular signals to modulate cell activation, differentiation and survival. In the hematopoietic system, it was demonstrated that gal-3 is expressed in myeloid cells and surrounding stromal cells. Furthermore, exogenous and surface gal-3 drive the proliferation of myeloblasts in a granulocyte-macrophage colony-stimulating factor (GM-CSF)-dependent manner. Here, we investigated whether gal-3 regulates the formation of myeloid bone marrow compartments by studying galectin- $3^{-/-}$mice $\left(\right.$gal- $3^{-/-}$) in the C57BL/6 background.
\end{abstract}

C. Brand $\cdot$ F. L. Oliveira $\cdot$ L. Ricon $\cdot$ L. C. Boldrini

R. Borojevic $\cdot$ M. Farina $\cdot$ M. C. El-Cheikh $(\square)$

Laboratório de Proliferação e Diferenciação Celular,

Instituto de Ciências Biomédicas,

Universidade Federal do Rio de Janeiro,

Av. Carlos Chagas Filho, 393,

CEP: 21941-902 Rio de Janeiro, RJ, Brazil

e-mail: marcia@histo.ufrj.br

\section{L. Fermino}

Laboratório de Glicobiologia e Imunoquímica - Departamento de Biologia Celular e Molecular e Bioagentes Patogênicos,

Faculdade de Medicina Universidade de São Paulo,

Ribeirão Preto, SP, Brazil

\section{K. Hsu • F. T. Liu}

Department of Dermatology, School of Medicine,

University of California Davis,

Sacramento, CA, USA

\section{R. Chammas}

Laboratório de Oncologia Experimental e Instituto do Câncer do Estado de São Paulo - Faculdade de Medicina, Universidade de São Paulo and Centre for Cell-Based Therapy CEPID-FAPESP, São Paulo, Brazil
The bone marrow histology of gal-3 $3^{-/}$mice was significantly modified and the myeloid compartments drastically disturbed, in comparison with wild-type (WT) animals. In the absence of gal-3, we found reduced cell density and diaphyseal disorders containing increased trabecular projections into the marrow cavity. Moreover, myeloid cells presented limited capacity to differentiate into mature myeloid cell populations in gal- $3^{-/-}$mice and the number of hematopoietic multipotent progenitors was increased relative to WT animals. In addition, bone marrow stromal cells of these mice had reduced levels of GM-CSF gene expression. Taken together, our data suggest that gal-3 interferes with hematopoiesis, controlling both precursors and stromal cells and favors terminal differentiation of myeloid progenitors rather than proliferation.

Keywords Hematopoiesis · Galectin-3 · Myeloid differentiation

\section{Introduction}

Galectin-3 (gal-3) is a highly promiscuous lectin detected in the extracellular matrix, on the cell surface and within the nucleus and cytoplasm, where it can regulate several biological systems (Henderson and Sethi 2009). In extracellular environments, gal-3 is capable of modulating inflammatory responses by favoring monocyte and neutrophil activation (Liu et al. 1995; Yamaoka et al. 1995). In parallel, galectin-3 null mice (gal- $3^{-/-}$mice) subjected to an acute and/or chronic inflammatory reaction exhibited a reduced influx of neutrophils and macrophages into the inflammatory site as well as a delayed capacity of monocyte-macrophage differentiation (Colnot et al. 1998; Hsu et al. 2000; Sano et al. 2000; Oliveira et al. 2007; 
Nieminen et al. 2008). Recently, we described the regulation of B1 and B2 lymphocyte differentiation into plasma cells in the bone marrow, peritoneal cavity and mesentery compartments by gal-3 (Oliveira et al. 2007, 2009).

Several biological molecules control specific stages of proliferation and differentiation of hematopoietic stem cells (HSCs) into mature blood cells inside the bone marrow (Weissman 2000; Sugiyama et al. 2006). The continuous production of cells of both lymphoid and the myeloid lineages depends on the correct spatial organization of putative HSCs and their progenies located in specific stromal cell niches and in constitutive contact with growth factors, such as granulocyte-macrophage colony-stimulating factor (GM-CSF). By controlling cell adhesion, motility and differentiation, extracellular matrix components such as glycosaminoglycans (Gallagher et al. 1983) and cell membrane components, including integrins and gangliosides, have been identified as important regulators in hematopoiesis (Andrade et al. 2006; Ziulkoski et al. 2009).

Although gal-3 is expressed by bone marrow cells, the role of the lectin-carbohydrate interaction during hematopoiesis is still unclear. In this context, it was shown that bone marrow cells alter the surface lectin patterns during the process of differentiation (Krugluger et al. 1994) and that endogenous gal-3 modified the GM-CSF-driven proliferation of immature myeloid cells (Krugluger et al. 1997). Moreover, it was also demonstrated that gal-3 was expressed in myeloblasts, mature myeloid cells and surrounding stromal cells, suggesting that this lectin is involved with the organization of the myeloid compartments (Krugluger et al. 1997). Recently, we described the presence of gal-3 in a well-defined B220 $0^{\text {high }}$ B cell subpopulation in the bone marrow and that in its absence, there is accelerated differentiation of B cells into plasma cells (Oliveira et al. 2007).

Here, we investigated whether myeloid subpopulations were modified in absence of gal-3, considering that both extracellular and surface gal-3 drive the proliferation and differentiation of hematopoietic cells in physiological and pathological conditions. Thus, we analyzed the bone marrow compartment in wild-type (WT) and gal- $3^{-/-}$mice.

\section{Materials and methods}

Mice

Inbred C57/BL6 (WT) mice and gal- $3^{-/-}$mice (backcrossed to C57/BL6 for 9 generations) (Hsu et al. 2000), age and sex matched, were obtained from the colony bred at the Federal University of Rio de Janeiro (Brazil). Mice manipulations were performed in accordance with institu- tional guidelines for the use and care of laboratory animals (protocol DAHEICB 009, Federal University of Rio de Janeiro, Brazil).

\section{Histological analysis of bone marrow}

For histological evaluation, intact femurs of WT and gal- $3^{-/}$ mice were surgically removed, carefully cleaned by mechanical procedures and immersed in a solution containing $2.5 \%$ glutaraldehyde and $4 \%$ formaldehyde freshly prepared from paraformaldehyde in $0.1 \mathrm{M}$ phosphate buffer for $24 \mathrm{~h}$. Afterwards, the samples were washed in the buffer, dehydrated in an acetone series from 30 to $100 \%$ ( $\mathrm{v} / \mathrm{v}$ in water), $15 \mathrm{~min}$ in each step and embedded in the Spurr resin (Ted Pella, Redding, CA, USA). Transverse semithin sections of circa $5 \mu \mathrm{m}$ from the femurs at the diaphysis region were obtained with a diamond knife in a Sorvall Porter-Blum MT2-B ultramicrotome and double stained with toluidine blue and basic fuchsin (SigmaAldrich, Saint Louis, MI, USA). The slides obtained were mounted using Entelan (Merck, Darmstadt, Germany) and analyzed in a Zeiss Axioplan (Oberkochen, Germany) light microscope in the bright-field mode. The micrographs obtained were digitalized with an Evolution MP 5.0 cooled CCD color camera (MedyaCybernetics, Bethesda, MD, USA). The cell density was performed by histomorphometric analyses using the ImageJ software in five fields of each sample imaged with a $\times 40$ objective.

\section{Bone marrow cell suspensions}

Bone marrow cells were obtained ex vivo by flushing the femoral cavity of WT and gal-3 $3^{-/-}$mice with phosphatebuffered saline (PBS), $\mathrm{pH} 7.2$, with $3 \%$ fetal bovine serum (FBS, LGC; Cotia, São Paulo, Brazil). Cells were washed, quantified by counting and prepared for further experimentation. When indicated, red blood cell lyses were done with hypotonic ACK (ammonium chloride potassium) solution.

Antibodies and flow cytometry analysis

In order to saturate $\mathrm{Fc}$ receptors, $1 \times 10^{6}$ cells $/ \mathrm{mL}$ bone marrow-derived cells were incubated with the $\mathrm{Fc}$ blocker antibody, produced by clone 2.4G2 (obtained from the Rio de Janeiro Cell Bank PABCAM, Federal University, Rio de Janeiro, Brazil), for 10 min before adding specific monoclonal antibodies (mAbs). Cells were incubated with mAbs for $30 \mathrm{~min}$; unbound $\mathrm{mAbs}$ were washed out with PBS. The following mAbs were used: FITC-labeled Sca-1, Mac-1 and IgM; PE-labeled CD34, Ter-119 and B220; PE Cy5.5labeled Gr-1 and Biotin-labeled $\mathrm{Lin}^{-}$and IgD; PerCPlabeled streptoavidin and APC-labeled c-Kit (all from BD Bioscience, San Jose, CA, USA). Samples were acquired in 
a flow cytometer (FACScalibur; BD Bioscience) using Cell Quest software and analyzed using WinMDI 2.9 software.

\section{Cell lines}

Primary cultures of M3/38, L929, MM3 and WeHi3B cells secreting anti-gal-3 antibody, macrophage colony stimulating factor (M-CSF), granulocyte-macrophage colony-stimulating factor (GM-CSF) and interleukin-3 (IL-3) respectively, were obtained from the Rio de Janeiro Cell Bank PABCAM, Federal University of Rio de Janeiro. The supernatants were harvested from confluent cultures, filtered, aliquoted and stored at $-20^{\circ} \mathrm{C}$ until used.

Cultures of bone marrow-derived cells

Soft-agar cultures Cell culture systems were prepared as described by Heyworth and Spooncer (1993). Briefly, total bone marrow-derived cells obtained from WT and gal- $3^{-/}$ mice were plated at $5 \times 10^{4} / \mathrm{ml}$ in double-layer soft-agar assays. The bottom layer was prepared at a $0.4 \%$ final agar concentration in Iscove's medium (Sigma-Aldrich) with $10 \%$ FBS, plated in 35-mm Petri dishes (Corning, New York, USA). The upper layer containing the cells $(0.33 \%$ final agar concentration) were supplemented either with $10 \%$ conditioned medium from MM3, L929 or WeHi3B cells.

Long-term cultures of bone marrow-derived cells Total bone marrow-derived cells of WT and gal- $3^{-1-}$ mice were plated in culture medium and, after $4 \mathrm{~h}$, the cultured cells were washed vigorously and the resting adherent cells evaluated. After 4 weeks in culture, the morphological and molecular essays were performed in the stromal cells.

Short-term cultures of bone marrow-derived cells Liquid cell cultures were performed with non-adherent bone marrow-derived cells of WT mice. To obtain non-adherent cells, total bone marrow was plated in a plastic dish of 60 $\mathrm{mm}$ for $1 \mathrm{~h}$ and nonadherent cells were cultured in 24-well culture plates (Corning) in $\alpha$-MEM (Sigma-Aldrich) supplemented with $5 \%$ of FBS in the presence of medium alone, in presence of $20 \%$ conditioned medium containing GM-CSF factor and with GM-CSF plus anti-gal-3 antibody. Part of the cells were harvested after $48 \mathrm{~h}$, quantified, centrifuged on glass slides in a cytospin, stained and analyzed morphologically. The cells were quantified as myeloid precursors, including those with the ring-form nucleus, polymorphonuclear neutrophils (PMN) and monocytes (El-Cheikh and Borojevic 1990). After $48 \mathrm{~h}$ of treatment with GM-CSF and GM-CSF plus anti-gal-3 antibody, the remaining cells were harvested and plated in methylcellulose medium (Methocult M3630; Stem Cell Technologies, Vancouver, Canada), to evaluate their capacity of proliferation.

For the semisolid culture system, such as soft-agar and methylcellulose, colonies (>50 cells) and clusters $(<50$ cells) were quantified after 8 days in an inverted microscope (Olympus CKX41SF; Shinjuku, Tokyo, Japan). Morphological analyses were confirmed in agar cultures fixed overnight with formol vapor, floated onto microscope slides, dried and stained with May-Grunwald and Giemsa (Sigma-Aldrich) and analyzed in a Zeiss-Axioplan, or an Olympus CKX41SF light microscope. The images of the clusters and colonies were acquired in the bright-field mode using a camera Evolution MP 5.0 RTV (Color-Media Cybernetics, Canada).

Real-time quantitative PCR analysis

After 1 week in culture, the non-adherent bone marrowderived cells were discarded and the adherent fraction was extracted. The total RNA was isolated by using the TRIzol reagent (Invitrogen Life Technologies, Carlsbad, CA, USA), following the manufacturer's instructions. cDNA synthesis was performed in a final volume of $20 \mu \mathrm{L}$, using ImProm-II reverse transcriptase (Promega, Madison, WI, USA). The reaction mixture contained $4 \mu \mathrm{g}$ total RNA, 20 pmol oligo dT primer (Invitrogen Life Technologies, San Diego, CA, USA), $40 \mathrm{U}$ RNasin, $500 \mu \mathrm{M}$ of dNTP mix and $1 \mathrm{U}$ reverse transcriptase in the $1 \times$ reverse transcriptase buffer. The cDNA was treated with $10 \mu \mathrm{g}$ RNase (Gibco, Carlsbad, CA, USA) and then immediately used or stored at $-20^{\circ} \mathrm{C}$. PCR amplification and analysis were done using an ABI Prism 7500 sequence detector (Applied Biosystems, Foster City, CA, USA). All the reactions were done with SYBR Green Master Mix (Applied Biosystems) using a 25$\mu \mathrm{L}$ volume in each reaction, which contained $2 \mu \mathrm{L}$ template cDNA, 5 pmol each primer and $12.5 \mu \mathrm{L}$ SYBR Green. The primers used for PCR amplification were $\beta$-actin (forward: 5-AGCTGCGTTTTACACCCTTT-3, reverse: 5-AAGCCATGCCAATGTTGTCT-3); GM-CSF (forward:5CCTGGGCATTGTGGTCTACAG-3, reverse:5-GGTTCA GGGCTTCTTTGATGG-3). The relative expression of GMCSF gene was obtained using the comparative CT method and was normalized using $\beta$-actin as an endogenous control. Data are expressed as relative gene expression ratios between mRNA levels of the target gene versus $\beta$-actin.

Statistical analysis

The statistical tests were accomplished using Tukey's multiple comparison test ( $t$ test) and the significance threshold was fixed for $\alpha=0.05$. Therefore, $P$ values $\leq 0.05$ were considered statistically significant. 


\section{Results}

Histological aspects of bone marrow

Considering that gal-3 is present in bone marrow compartments, we first performed histological analysis of the femural diaphysis in both WT and gal- $3^{-1-}$ mice (Fig. 1a and b, respectively). As previously described (Weiss and Geduldig 1991; Bianco et al. 2001; Sacchetti et al. 2007), we noted that bone marrow of WT mice had a classic diaphyseal organization, containing a high cellular density and poorly infiltrated with trabecular projections. Moreover, we confirmed that the subendosteal microenvironment was continuously connecting the bone surface and densely packaged hematopoetic cells (Fig. 1a, c). On the other hand, the bone marrow compartment of gal- $3^{-/}$ mice showed a lower cellular density and a higher quantity of trabecular projections in the marrow cavity (Fig. 1b, d).
Megakaryocytes that are normally located on the central region of the bone cavity (Fig. 1c) were frequently present surrounding the endosteum in these knockout mice (Fig. 1d, arrow). A significant lower cellularity was also observed in the bone marrow of gal- $3^{-/}$mice (Fig. 1e). Together, these data suggested that structural changes in the architecture of the bone marrow in absence of gal-3 can be associated with homeostatic systems that control the cell density within the bone marrow.

Absence of galectin-3 modifies the pattern of precursor cells inside the bone marrow

Hematopoiesis is a well-regulated process that depends on the correct distribution of different precursors and their progenies and on the equilibrium between stem cells, committed progenitors and mature cells (Weissman 2000; Sugiyama et al. 2006). Considering that the bone marrow of
Fig. 1 Bone marrow/endosteum interface of wild type (WT) and in gal- $3^{-/-}$mice, at a diaphysis region near the metaphysis. a) Longitudinal section of samples from WT mice showing a continuous and smooth contour of the endosteum region and normal appearance of the marrow cavity with a homogeneous distribution of hematopoetic cells. b Longitudinal section of a region similar to the previous figure from a femur of a gal- $3^{-l}$ mice. In this case, a lower density was observed as well as the presence of projections of bone tissue (insert) in the bone marrow cavity. c, d Detailed views of regions described in (a) and (b). In gal- $3^{-/-}$mice (d), megakaryocytes attached to the endosteum region were observed (arrows). e Quantification of the number of cells per field for WT and in gal- $3^{--}$mice. Data are reported as mean $\pm \mathrm{SD}$ and are representative of three independent experiments, each one carried out with three WT and gal- $3^{-/-}$mice. Statistical analysis: Tukey's multiple comparisons test $(*, P<0.05)$. All sections were stained with toluidine-blue followed by basic fuchsin

gal- $3^{-/-}$mice was empty, we quantified by flow cytometry the different cell populations in both experimental groups. The results described here are expressed in percent instead of the absolute numbers, since bone fragmentation procedures to perform flushing can influence total cell yields. There was no statistical difference in the number of hematopoietic multipotent progenitors $\left(\mathrm{Lin}^{-}, \mathrm{Sca}-1^{+}\right.$, c$\mathrm{Kit}^{+}, \mathrm{CD} 34^{-}$cells) that contain the putative hematopoietic stem cells with a capacity for long-term bone marrow repopulation when WT and gal- $3^{-/-}$mice were compared (Fig. 2a). However, the number of hematopoietic multipotent progenitors $\left(\mathrm{Lin}^{-}, \mathrm{Sca}-1^{+}, \mathrm{c}-\mathrm{Kit}^{+}, \mathrm{CD} 34^{+}\right.$cells), including cells with short-term capacity to repopulate a bone marrow, was increased in $\mathrm{gal}-3^{-/}$mice when compared with WT mice (Fig. 2b). Related to hematopoietic cascade of cell differentiation, we analyzed downstream progenitors: common lymphoid progenitors (CLPs, $\mathrm{Lin}^{-}$, Sca-1 ${ }^{\text {low }}, \mathrm{c}-\mathrm{Kit}^{\text {low }}, \mathrm{CD} 34^{-}$) and common myeloid

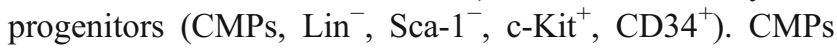
were increased in the bone marrow of gal- $3^{-1-}$ mice (Fig. 2c) but no difference was observed as to CLPs numbers in both groups (Fig. 2d). There was no significant differences observed in megakaryocytic/erythroid progenitors (MEPs, $\mathrm{Lin}^{-}, \mathrm{Sca}^{-}{ }^{-}, \mathrm{c}^{-\mathrm{Kit}^{+}}, \mathrm{CD} 34^{-}$) (Fig. 2f) and in erythroid progenitors (Ter $119^{+}$) (data not shown).

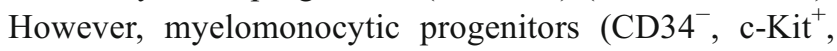
Mac- $\left.1^{+}, \mathrm{Gr}_{-}{ }^{+}\right)$, mature granulocytes $\left(\mathrm{CD} 34^{-}, \mathrm{c}^{-\mathrm{Kit}^{-}}\right.$, Mac- $\left.1^{+}, \mathrm{Gr}-1^{\text {high }}\right)$ and monocytes $\left(\mathrm{CD} 34^{-},{\mathrm{c}-\mathrm{Kit}^{-}}^{-}\right.$, Mac$\left.1^{+}, \mathrm{Gr}-1^{\text {low }}\right)$ were significantly reduced in absence of gal-3 (Fig. 2e, g and h, respectively). These data obtained from phenotypic analyses suggested that in the absence of gal-3 the bone marrow compartment is altered toward an increase of hematopoietic multipotent progenitors with a low capacity to differentiate in mature myeloid and monocyte cell populations.

In order to access the proliferate capacity of these myeloid progenitors in the bone marrow of gal- $3^{-/-}$mice, we performed cell culture experiments using conditioned semisolid substrate (soft-agar) and monitored the growth of cell colonies and clusters. After 1 week, the number and size of both clusters and colonies were significantly increased in samples obtained from bone marrow of gal$3^{-/-}$mice (Fig. 3a and b, respectively). In the morphological analysis performed in the colonies, we observed a prevalence of immature and mature polymorphonuclear neutrophils in colonies derived from WT mice (Fig. 3c, insert). In contrast, mononuclear monocyte-like cells were predominant in colonies obtained from gal- $3^{-/}$mice (Fig. 3d, insert). Considering the increased number and size of clusters and colonies after in vitro stimuli, we analyzed the blood of these mice to quantify neutrophils and monocytes. In fact, there was a neutropenia in the blood of these gal- $3^{-/-}$mice (Fig. 3e) without modification


h



Fig. 2 Phenotypic characterization of bone marrow myeloid cells in WT (solid bars) and gal- $3^{-/-}$mice (open bars). a Long-term hematopoietic multipotent progenitors (LT-HMP, $\mathrm{Lin}^{-}, \mathrm{Sca}^{-}{ }^{+}$, c$\mathrm{Kit}^{+}, \mathrm{CD}^{-} 4^{-}$). (b) Short-term hematopoietic multipotent progenitors


progenitors (CMPs, $\mathrm{Lin}^{-}, \mathrm{Sca}^{-}{ }^{-},{\mathrm{c}-\mathrm{Kit}^{+}}^{+}, \mathrm{CD} 34^{+}$). (d) Common lymphoid precursors (CLP, $\mathrm{Lin}^{-}, \mathrm{Sca}-1^{\text {low }}, \mathrm{c}-K i t^{\text {low }}, \mathrm{CD} 34^{-}$). (e) Myelomonocytic progenitors (MMPs, CD34 ${ }^{-}, \mathrm{c}-K i t^{+}, \mathrm{Mac}^{-}{ }^{+}, \mathrm{Gr}-1^{+}$). (f) Megakaryocytic/erytrhoid progenitors (MEPs, $\mathrm{Lin}^{-}, \mathrm{Sca}-1^{-}, \mathrm{c}^{-} \mathrm{Kit}^{+}$, CD34- $)$. (g) Granulocytes (Mac-1 $\left.{ }^{+}, \mathrm{Gr}-1^{\text {high }}\right)$. (h) Monocytes $\left(\right.$ Mac- $^{+}$, $\left.\mathrm{Gr}-1^{\text {low }}\right)$. The data were obtained from three independent experiments carried out with three WT and gal- $3^{-/-}$mice. Statistical analysis: Tukey's multiple comparison test $(*, P \leq 0.05)$ 
Fig. 3 Numbers of colonyforming units (CFU) in soft-agar clonogenic assays in $5 \times 10^{4} / \mathrm{ml}$ bone marrow cells from WT and gal- $3^{-/}$mice. a Number of clusters ( $\leq 50$ cells) and $\mathbf{b}$ colonies $(\geq 50$ cells) analyzed at 8 days of culture. c The size and the predominant cell type in the colonies from WT (insert in granulocytes) and $\mathbf{d}$ in gal- $3^{-/-}$mice cultures (insert in mononuclear cells). e Quantification of neutrophils in blood smears of WT and gal- $3^{-/-}$mice. f Quantification of monocytes in blood smears of WT and gal- $3^{-/-}$mice. Results represent mean values and standard errors of three independent experiments using three WT and gal- $3^{-/-}$mice. Statistical analysis: Tukey's multiple comparison test $(*, P<0.05)$
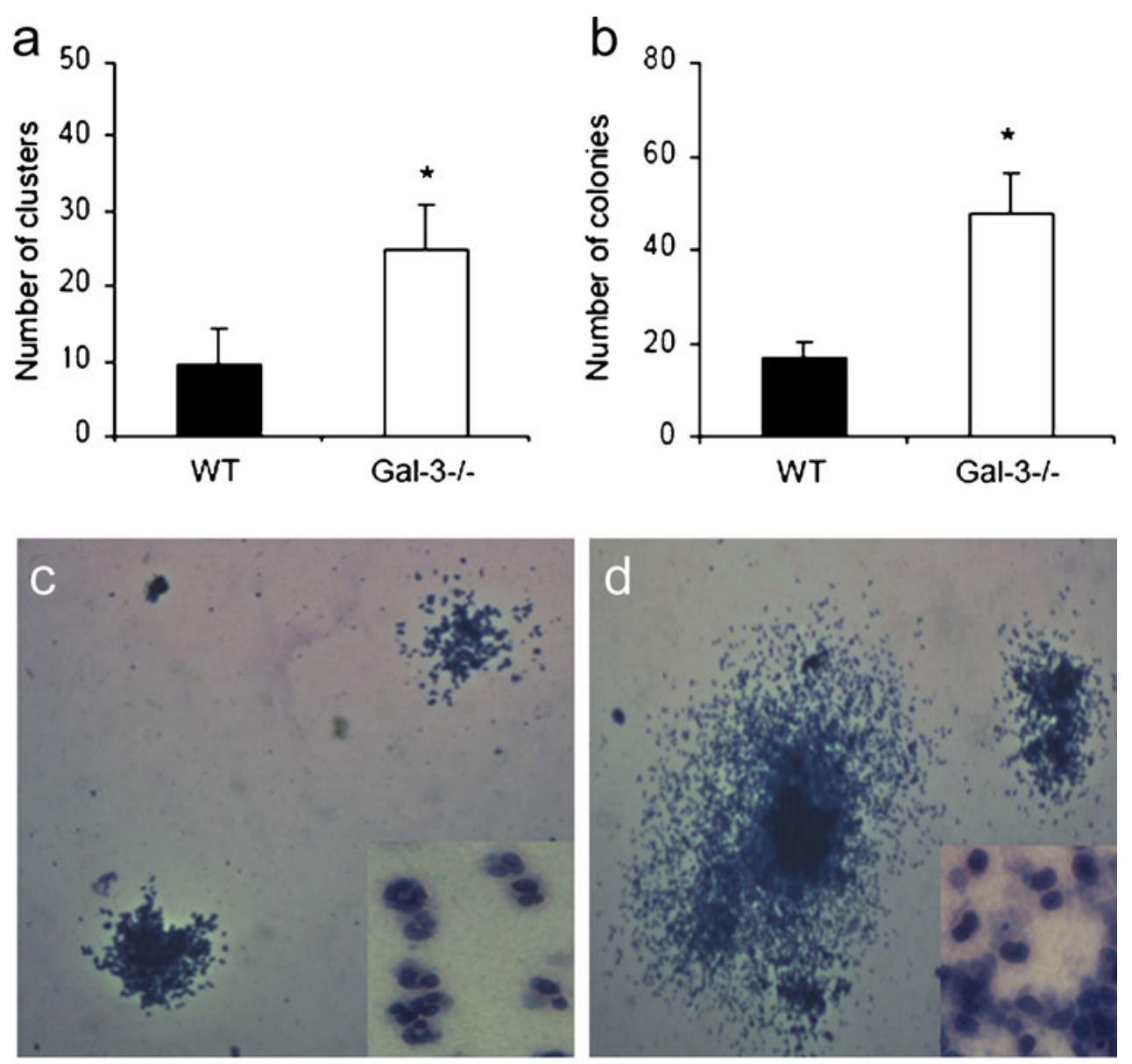

e

Neutrophils

f
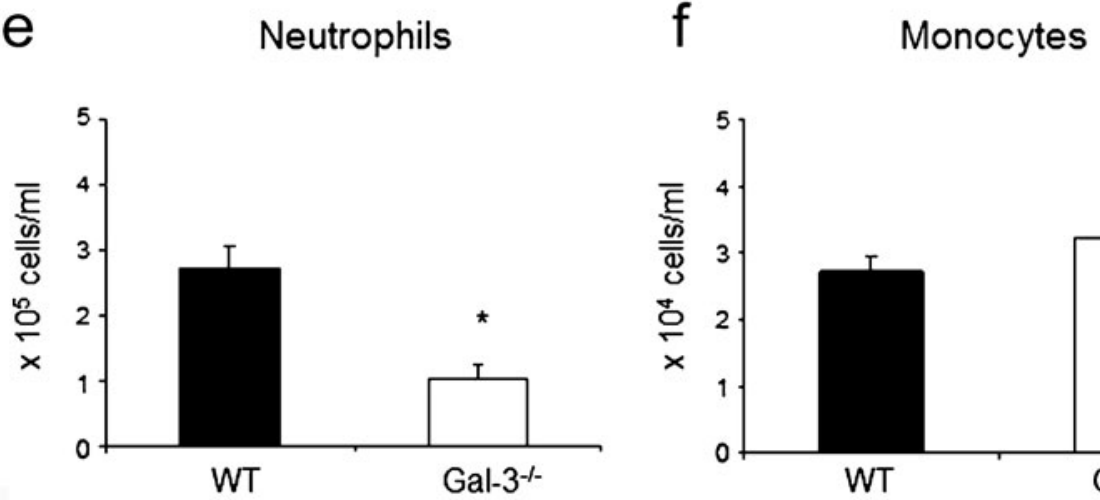

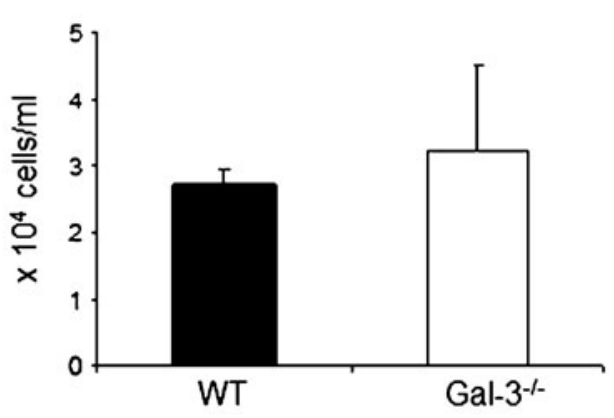

in monocyte numbers (Fig. 3f), in contrast to the results obtained in the bone marrow (Fig. 2h).

Absence of galectin-3 altered the behavior of bone marrow stromal cells in vitro

The long-term bone marrow culture system (LBMC) is an in vitro model that permits the study of hematopoietic and stromal cell interactions in a time-dependent manner since stem cells and progenitors cells can be maintained for extended periods in culture (Dexter et al. 1977). In order to evaluate if the myeloid disturbance observed in gal- $3^{-/-}$mice could be related to stromal defects, we cultured bone marrow-derived cells in long-term conditions. After $48 \mathrm{~h}$ of cell culture, a well-defined monolayer in both groups was observed. However, hematopoietic cells of gal- $3^{-1-}$ mice were predominantly organized in clusters (Fig. 4b) when compared with WT samples (Fig. 4a). After 4 weeks, when the stromal feeder layer was established, we observed significant morphological differences in reticular cells, when compared with WT and gal- $3^{-1-}$ mice (Fig. 4c and d, respectively). In the long-term cultures, the reticular stromal 
Fig. 4 The long-term bone marrow cell cultures and molecular assay. The adherent bone marrow-derived cells of WT (a) and gal- $3^{-/-}$mice (b) were maintained in culture for $48 \mathrm{~h}$. The hematopoietic cells from gal- $3^{-1-}$ mice proliferate more in vitro as demonstrated by the presence of clusters in culture (arrowhead). After 4 weeks, the cells of gal- $3^{-/}$ mice (d) in culture presented cobblestone areas (white arrow) and stromal cells accumulating lipids droplets (black arrow) compared with WT mice cells in culture (c). e Levels of mRNA expression of GM-CSF in $10^{6}$ adherent cells obtained from bone marrow of WT (solid bar) and gal- $3^{-/}$mice (open bar) after 4 weeks of culture. Results represent mean values and standard errors of three independent experiments using three WT and gal- $3^{-/-}$mice. Statistical analysis: Tukey's multiple comparison test $(*, P<0.05)$
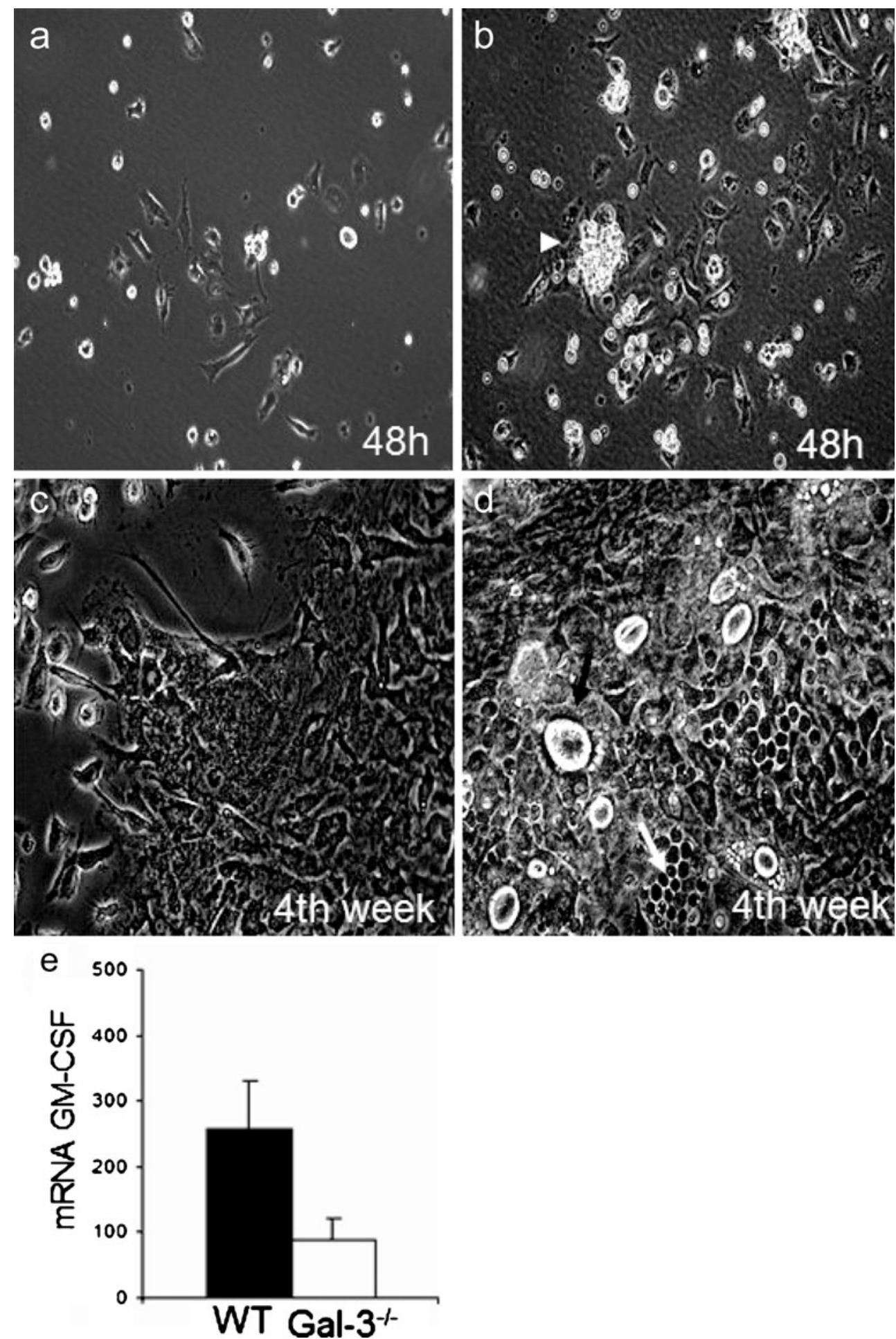

cells of gal- $3^{-/}$mice presented as a blanket of cells residing over hematopoietic cells, forming cobblestone areas and accumulating lipid droplets (Fig. 4d, arrow). It is important to note that rare stromal cells from WT mice accumulated lipids and the cobblestone areas were not observed (Fig. 4c). As these morphological changes could be associated with disturbed myelopoiesis and neutropenia, we monitored and found a significant reduction in mRNA GM-CSF levels expressed by stromal cells from bone marrow of gal- $3^{-/-}$ mice (Fig. 4e). Together, these data suggest that gal-3 participates in the regulation of hematopoiesis by stromal cells, partly by influencing GM-CSF gene expression. 
Galectin-3 modifies GM-CSF-driven bone marrow myeloid cell differentiation

Short-term bone marrow cell culture is an excellent in vitro system that permits evaluation of the terminal steps of cell differentiation in response to specific hematopoietic growth factors in a stroma-independent manner. In order to investigate whether gal-3 and GM-CSF are both involved in terminal differentiation of myeloid cells, we performed short-term culture of bone marrow-derived cells from WT mice and treated part of these cells with anti-gal-3 antibody. After $48 \mathrm{~h}$ in contact with exogenous GM-CSF, committed myeloid precursors differentiated into mature polymorphonuclear neutrophils (Fig. 5a). However, when the bone marrow-derived cells were treated with anti-gal-3 antibody in the presence of GM-CSF, the myeloid progenitors cells with typical ring nuclei outnumbered mature polymorphonuclear neutrophils (Fig. 5b). In fact, the treatment with GM-CSF induced a significant increase in the absolute number of the polymorphonuclear neutrophils and monocytes in vitro (Fig. 5c). On the other hand, a part of the myeloid cells remained as progenitor cells and the addition GM-CSF was not sufficient to induce the proliferation and differentiation into mature neutrophils and monocytes (Fig. 5c). Together, these data indicate that the partial inhibition of gal-3 in vitro increased the proliferative capacity of myeloid progenitor cells instead of differentiation into neutrophils and monocytes cells, corroborating the in vivo data.

In order to confirm the proliferative capacity of these myeloid precursors generated in the short-term culture system, the remaining non-adherent cells were cultured in the semisolid medium (methylcellulose) for 1 week and clusters and colonies were quantified. Cells pre-treated with GM-CSF alone promptly responded to growth factors in methylcellulose medium and formed well-defined colonies (Fig. 6a). However, cells pre-treated with GM-CSF and anti-gal-3 antibody were more responsive and showed an increased proliferation capacity (Fig. 6b). In fact, the number of colonies and clusters were significantly increased when these hematopoietic progenitor cells from WT mice were treated with GM-CSF and anti-gal-3 antibody (Fig. 6c and d, respectively). No colonies or clusters were observed in cultured cells obtained $48 \mathrm{~h}$ in medium supplemented only with fetal bovine serum (data not shown). These data permitted us to propose that gal-3 regulates myeloid cell proliferation/differentiation steps in a GM-CSF-dependent manner. The data obtained in vivo and in vitro reinforce the role of gal-3 on the myeloid bone marrow compartment.
Fig. 5 Morphological analysis and counts of non-adherent hematopoietic cells of WT mice in short-term bone marrow culture. a Cells stimulated with GM-CSF and $\mathbf{b}$ cells stimulated with GM-CSF and treated with anti-gal-3. c Differential counts of myeloid precursors (arrowhead in b), (PMN) polymorphonuclear neutrophils (arrow in a) and monocytes in control group (medium supplemented with FCS), GM-CSF stimulated and GM-CSF plus anti-gal-3 treated cells. Results represent mean values and standard errors of three independent experiments using three WT mice. Statistical analysis: Tukey's multiple comparison test $(*, P<0.05)$




Fig. 6 Clonogenic assay of non-adherent hematopoietic cells of WT mice after $48 \mathrm{~h}$ in culture in the presence of GM-CSF (a) and GM-CSF plus anti-gal-3 antibody (b), performed in methylcellulose medium for 1 week. The colonies $(>50$ cells) $(\mathbf{c})$ and clusters $(<50$ cells $)($ d) were quantified in cultures of cells previously treated with GM-CSF (solid bar) and with GM-CSF plus anti-gal-3 antibody (open bar). Results represent mean values and standard errors of two independent experiments using WT mice. Statistical analysis: Tukey's multiple comparison test $\left(^{*}, P<0.05\right)$
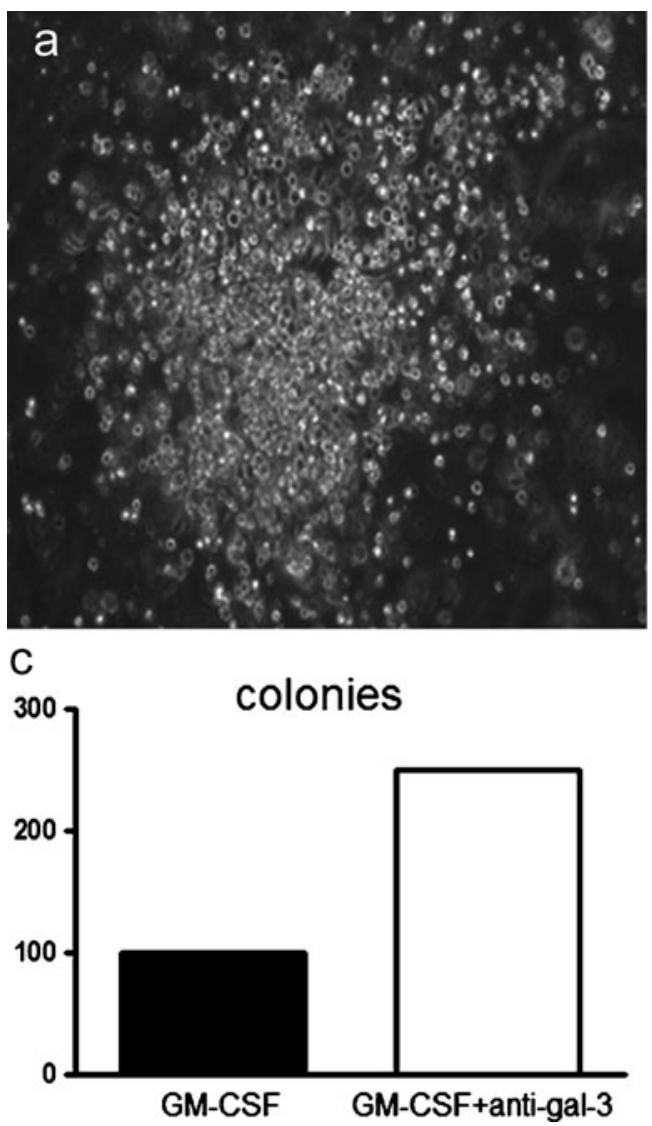



d



\section{Discussion}

Our results obtained in vivo and in vitro show alterations in the bone marrow compartment of gal- $3^{-1-}$ mice. The femoral diaphysis in these mice was intensely trabecular, containing a significant infiltrate of the bone tissue inside the medullar cavity contributing to a spongy fragility and increased interface between bone tissue and hematopoietic cells. The fragility of femurs was observed in the flushing procedures to obtain bone marrow cells, when femurs of gal- $3^{-/-}$mice were more easily fragmented than the controls (personal observation, data not shown).

Dominici and colleagues (2009) demonstratated that, after bone marrow irradiation, the megakaryocytes leave their vascular niches and migrate to the endosteal surface, where osteoblasts secrete large amounts of SDF-1, responsible for their homing and survival. In gal- $3^{-/}$mice, we observed reduced cell density in the marrow cavity, although less drastic than that observed in bone marrow ablation but an abnormal subendosteal localization of megakaryocytes. Thus, the physical dislodged of megakaryocytes in bone marrow could be a consequence of disturbance in the production of homing factors (Dominici et al. 2009) as well as in the structural organization of the bone marrow, or both.

In bone development, gal-3 is found in osteoblasts, osteoclasts and chondrocytes (Aubin et al. 1995; Colnot et al. 1999). In addition, gal-3 is implicated in chondrocyte death and vascular invasion altering the chondrogenesis process (Colnot et al. 2001). The histological disturbance observed in the bone marrow of gal- $3^{-/-}$mice can be a consequence of the abnormal chondrogenesis (Colnot et al. 2001) and studies in the earlier phases of bone development could explain the relationships between the ossification process and hematopoiesis. Moreover, these improperly located trabeculae could promote rearrangement of bone cells, such as osteoblasts and osteoclasts, favoring the displacement of hematopoietic niches. These arguments corroborate with our findings in the bone marrow of gal- $3^{-/-}$mice, where we detected increased numbers of multipotent hematopoietic and common myeloid precursors.

Here, histological data also showed lower cell populations in the diaphyseal bone cavity in gal- $3^{-1-1}$ mice, where we found that the number of granulocytes $\mathrm{Mac}-1^{+} \mathrm{Gr}-1^{\text {high }}$ and monocytes/macrophages $\mathrm{Mac}-1^{+} \mathrm{Gr}-1^{\text {low/neg }}$ was significantly reduced, thus explaining this moderate aplasia. In contrast, the in vitro assays performed revealed a high proliferation potential of myeloid progenitor cells and a reduced expression of GM-CSF in stromal cells. These results support the idea that these cells were not differentiating normally in vivo due to lower levels of GM-CSF present in the bone marrow compartment but they responded readily when this growth factor was added to 
the culture system as we demonstrated in vitro. In this context, Krugluger and colleagues demonstrated that extracellular gal-3 is restricted to the myeloid environments especially on the cell surface of precursors and mature myeloid cells (Krugluger et al. 1997). These authors also demonstrated that gal-3 suppressed GM-CSFinduced proliferation of bone marrow cells, suggesting that this lectin regulates myelopoietic environments, possibly controlling the availability of specific growth factors (Krugluger et al. 1997). Our results demonstrated that gal-3 indeed regulates the expression of GM-CSF at the stromal level, favoring the maintenance of precursors instead of myeloid cell differentiation.

The predominance of precursors in bone marrow of gal- $3^{-/-}$ mice is validated in vitro when the adherent cells provided a microenvironment to maintain the precursors for more time in culture (Prockop 1997). The long interactions between precursors and adherent cells could induce structural and functional modification in the adherent cells, such as decreased expression of GM-CSF, presence of blanket cells with cobblestone appearance and more fat-storing cells, as observed in the long-term cultures in the absence of gal-3.

Altogether, these results suggest that in the absence of gal-3, GM-CSF is not available to maintain an equilibrium between proliferation versus differentiation of myeloid cells. This hypothesis was confirmed in the experiments where gal-3 was blocked by anti-gal-3 antibody. In fact, the bone marrow-derived cells differentiated partially into segmented neutrophils, thus reproducing the data described in vivo. As gal-3 binds to the cell surface and controls the response of myeloid cells to GM-CSF, we propose that gal3 favors differentiation rather than proliferation of these cells. In B1 and B2 lymphocytes, gal-3 regulates the differentiation of these cells into plasma cells (Oliveira et al. 2009). These results suggest an important role of gal-3 on the cell membrane in the control of cell differentiation cascades.

Gal-3 is involved in neutrophil activation and adhesion (Kuwabara and Liu 1996), chemoattraction of monocytes/ macrophages (Sano et al. 2000) and opsonization of apoptotic neutrophils (Karlsson et al. 2009). The classical study done by Colnot et al. (1998), which demonstrated a delay in neutrophil recruitment into the peritoneal cavity after inflammatory stimulus in gal- $3^{-/-}$mice, could also be explained by the decrease of mature neutrophils inside of the bone marrow and not only by a defect in their initial recruitment. In this study, the authors discarded apoptosis as a mechanism to explain the delay in neutrophil recruitment (Colnot et al. 1998). Here, we propose a new function for gal-3 in neutrophil biology, since the lack of this lectin delayed neutrophil differentiation.

Gal-3 is defined as an important marker of monocyte differentiation into macrophages (Liu et al. 1995). As described here, myelomonocitic precursors and monocytes were decreased in the bone marrow of gal- $3^{-/}$mice followed by the reduction in peripheral blood and inflammatory tissue (Oliveira et al. 2007). Thus, we consider that gal-3 is crucial at the bone marrow level in the process of differentiation of myelomonocitic precursors to monocytes, although the quantity of common myeloid progenitor cells was superior in gal- $3^{-/-}$mice. The lower number of monocytes available in the marrow could alter the kinetics of mobilized inflammatory cells during the Schistosoma mansoni infection, interfering with the organization and collagen distribution in hepatic granulomas (Oliveira et al. 2007).

In the last decade, several hematological diseases had been characterized by hypocellularity in the bone marrow, reflected in a peripheral blood pancytopenia, such as aplastic anemia (Gaman et al. 2009). Preferentially, these hematological pathologies are associated with bone marrow stromal cell disorders that disturb the hematopoetic system. In addition to hypocellularity and blood neutropenia, fat cell hyperplasia, residual lymphocytosis, plasmocytosis and mastocytosis are common in the bone marrow of these experimental models or human patients that receive immunosuppressive treatment and additional hematopoietic growth factors, such as GM-CSF (Socie et al. 2007; Gaman et al. 2009). Some of these characteristics are observed in gal- $3^{-/-}$mice. However, as the bone marrow of these mice accumulates myeloid progenitors and shows delay in differentiation into mature myeloid cells, we suggested that gal-3 should be a critical molecule in the interface between physiological and pathological states.

In summary, we proposed a new role of gal-3 in early stages of myelopoiesis, delaying the process of differentiation via modulation of GM-CSF both in stromal and hematopoietic progenitors cells and a possible target for future investigations in hematological disorders, such as anemia and leukemia. The molecular mechanisms involved in the role of gal-3 in the bone marrow myelopoiesis are under evaluation.

Open Access This article is distributed under the terms of the Creative Commons Attribution Noncommercial License that permits any noncommercial use, distribution and reproduction in any medium, provided the original author(s) and source are credited.

\section{References}

Andrade CM, Ziulkoski AL, dos Santos AX, Boldrini LC, Trindade VM, Borojevic R, Guma FC (2006) Gangliosides of the stroma layer participate in the interferon-gamma receptor-dependent controls of myelopoiesis. Biochim Biophys Acta 1761:1030-1040 
Aubin JE, Liu F, Malaval L, Gupta AK (1995) Osteoblast and chondroblast differentiation. Bone 17:77S-83S

Bianco P, Riminucci M, Gronthos S, Robey PG (2001) Bone marrow stromal stem cells: nature, biology, and potential applications. Stem Cells 19:180-192

Colnot C, Ripoche MA, Milon G, Montagutelli X, Crocker PR, Poirier F (1998) Maintenance of granulocyte numbers during acute peritonitis is defective in galectin-3-null mutant mice. Immunology 94:290-296

Colnot C, Sidhu SS, Poirier F, Balmain N (1999) Cellular and subcellular distribution of galectin-3 in the epiphyseal cartilage and bone of fetal and neonatal mice. Cell Mol Biol (Noisy-le-Grand) 45:1191-1202

Colnot C, Sidhu SS, Balmain N, Poirier F (2001) Uncoupling of chondrocyte death and vascular invasion in mouse galectin 3 null mutant bones. Dev Biol 229:203-214

Dexter TM, Allen TD, Lajtha LG (1977) Conditions controlling the proliferation of haemopoietic stem cells in vitro. J Cell Physiol 91:335-344

Dominici M, Rasini V, Bussolari R, Chen X, Hofmann TJ, Spano C, Bernabei D, Veronesi E, Bertoni F, Paolucci P, Conte P, Horwitz EM (2009) Restoration and reversible expansion of the osteoblastic hematopoietic stem cell niche after marrow radioablation. Blood 114:2333-2343

El-Cheikh MC, Borojevic R (1990) Extramedullar proliferation of eosinophil granulocytes in chronic schistosomiasis mansoni is mediated by a factor secreted by inflammatory macrophages. Infect Immun 58:816-821

Gallagher JT, Spooncer E, Dexter TM (1983) Role of the cellular matrix in haemopoiesis. I. Synthesis of glycosaminoglycans by mouse bone marrow cell cultures. J Cell Sci 63:155-171

Gaman A, Gaman G, Bold A (2009) Acquired aplastic anemia: correlation between etiology, pathophysiology, bone marrow histology and prognosis factors. Rom J Morphol Embryol 50:669-674

Henderson NC, Sethi T (2009) The regulation of inflammation by galectin-3. Immunol Rev 230:160-171

Heyworth CM, Spooncer E (1993) In vitro clonal assays for murine multipotential and lineage restricted myeloid cells. In: Testa NG, Molineux G (eds) Haemapoiesis. A practical approach. Oxford University Press, Oxford, pp 37-53

Hsu DK, Yang RY, Pan Z, Yu L, Salomon DR, Fung-Leung WP, Liu FT (2000) Targeted disruption of the galectin-3 gene results in attenuated peritoneal inflammatory responses. Am J Pathol 156:1073-1083

Karlsson A, Christenson K, Matlak M, Bjorstad A, Brown KL, Telemo E, Salomonsson E, Leffler H, Bylund J (2009) Galectin-3 functions as an opsonin and enhances the macrophage clearance of apoptotic neutrophils. Glycobiology 19:16-20

Krugluger W, Koller M, Allmaier M, Boltz-Nitulescu G, Forster O (1994) Ligation of $\mathrm{N}$-acetylgalactosamine-containing structures on rat bone marrow cells enhances myeloid differentiation and murine granulocyte-macrophage colony-stimulating factorinduced proliferation. J Leukoc Biol 55:127-132

Krugluger W, Frigeri LG, Lucas T, Schmer M, Forster O, Liu FT, Boltz-Nitulescu G (1997) Galectin-3 inhibits granulocyte- macrophage colony-stimulating factor (GM-CSF)-driven rat bone marrow cell proliferation and GM-CSF-induced gene transcription. Immunobiology 197:97-109

Kuwabara I, Liu FT (1996) Galectin-3 promotes adhesion of human neutrophils to laminin. J Immunol 156:3939-3944

Liu FT, Hsu DK, Zuberi RI, Kuwabara I, Chi EY, Henderson WR Jr (1995) Expression and function of galectin-3, a beta-galactosidebinding lectin, in human monocytes and macrophages. Am J Pathol 147:1016-1028

Nieminen J, St-Pierre C, Bhaumik P, Poirier F, Sato S (2008) Role of galectin-3 in leukocyte recruitment in a murine model of lung infection by Streptococcus pneumoniae. J Immunol 180:24662473

Oliveira FL, Frazao P, Chammas R, Hsu DK, Liu FT, Borojevic R, Takiya CM, El-Cheikh MC (2007) Kinetics of mobilization and differentiation of lymphohematopoietic cells during experimental murine schistosomiasis in galectin-3 -/- mice. J Leukoc Biol $82: 300-310$

Oliveira FL, Chammas R, Ricon L, Fermino ML, Bernardes ES, Hsu DK, Liu FT, Borojevic R, El-Cheikh MC (2009) Galectin-3 regulates peritoneal B1-cell differentiation into plasma cells. Glycobiology 19:1248-1258

Prockop DJ (1997) Marrow stromal cells as stem cells for nonhematopoietic tissues. Science 276:71-74

Sacchetti B, Funari A, Michienzi S, Di Cesare S, Piersanti S, Saggio I, Tagliafico E, Ferrari S, Robey PG, Riminucci M, Bianco P (2007) Self-renewing osteoprogenitors in bone marrow sinusoids can organize a hematopoietic microenvironment. Cell 131:324-336

Sano H, Hsu DK, Yu L, Apgar JR, Kuwabara I, Yamanaka T, Hirashima M, Liu FT (2000) Human galectin-3 is a novel chemoattractant for monocytes and macrophages. J Immunol $165: 2156-2164$

Socie G, Mary JY, Schrezenmeier H, Marsh J, Bacigalupo A, Locasciulli A, Fuehrer M, Bekassy A, Tichelli A, Passweg J (2007) Granulocyte-stimulating factor and severe aplastic anemia: a survey by the European Group for Blood and Marrow Transplantation (EBMT). Blood 109:2794-2796

Sugiyama T, Kohara H, Noda M, Nagasawa T (2006) Maintenance of the hematopoietic stem cell pool by CXCL12-CXCR4 chemokine signaling in bone marrow stromal cell niches. Immunity 25:977-988

Weiss L, Geduldig U (1991) Barrier cells: stromal regulation of hematopoiesis and blood cell release in normal and stressed murine bone marrow. Blood 78:975-990

Weissman IL (2000) Stem cells: units of development, units of regeneration, and units in evolution. Cell 100:157-168

Yamaoka A, Kuwabara I, Frigeri LG, Liu FT (1995) A human lectin, galectin-3 (epsilon bp/Mac-2), stimulates superoxide production by neutrophils. J Immunol 154:3479-3487

Ziulkoski AL, dos Santos AX, Andrade CM, Trindade VM, Daniotti JL, Borojevic R, Guma FC (2009) Anchored and soluble gangliosides contribute to myelosupportivity of stromal cells. Biochem Biophys Res Commun 388:17-20 